\title{
Tantalizing New Physics from the Cosmic Purview
}

\author{
Fulvio Melia ${ }^{1}$ \\ Department of Physics, The Applied Math Program, and Department of Astronomy, The \\ University of Arizona, AZ 85721, USA. E-mail: fmelia@email.arizona.edu \\ ${ }^{1}$ John Woodruff Simpson Fellow.
}

\begin{abstract}
The emergence of a highly improbable coincidence in cosmological observations speaks to a remarkably simple cosmic expansion. Compelling evidence now suggests that the Universe's gravitational horizon, coincident with the better known Hubble sphere, has a radius improbably equal to the distance light could have travelled since the Big Bang. The confirmation of this unexpected result would undoubtedly herald the influence of new physics, yet appears to be unavoidable after a recent demonstration that the Friedmann-LemaitreRobertson-Walker metric is valid only for the so-called zero active mass equation of state. As it turns out, a cosmic fluid with this property automatically produces the aforementioned equality, leaving little room for a cosmological constant. The alternative - a dynamical dark energy — would suggest an extension to the standard model of particle physics, and a serious re-evaluation of the Universe's early history.
\end{abstract}

PCAC numbers: 04.20.Jb, 95.30.Sf, 98.80.Es, 98.80.Jk

\section{Introduction}

As cosmological observations stretch our view of the Universe to progressively higher redshifts, allowing us to probe physics at the earliest moments after the Big Bang, general relativity (GR) reminds us to be wary of limits to our exploration arising from the possible influence of various horizons. In cosmology, the term 'horizon' has been used to characterize the maximum distance particles could have traveled since the beginning - the observer's 'particle horizon' - or an imaginary surface that forever separates spacetime events that are causally connected to each other from those that are not - the 'event horizon' [1]. Several other definitions have a more customized application, such as the 'acoustic horizon', characterizing the maximum comoving distance traveled by sound waves prior to recombination, giving rise to the sub-degree scale anisotropies seen in the cosmic microwave background (CMB).

But it is another type of horizon, less familiar than these, that will command much of our attention in this paper. Known as an 'apparent horizon' in GR, this membrane may emerge in general, spherically or non-spherically symmetric spacetimes, subdividing congruences of ingoing and outgoing null geodesics from a given compact region. In the case of spherical geometry, these are just the ingoing and outgoing radial null geodesics from a two-sphere of symmetry $[2,3,4,5]$.

Indeed, for a spherically-symmetric spacetime, such as the Friedmann-Lemaittre-RobertsonWalker (FLRW) metric used extensively in modern cosmology, the apparent horizon may be 
understood more readily when characterized as a gravitational horizon, with a radius $R_{\mathrm{h}}$ thatin this context - coincides with the size of the better known Hubble sphere [6]. In fact, we now know that the Hubble radius owes its existence to these gravitational effects. In retrospect, the role played by the $R_{\mathrm{h}}$ horizon in our interpretation of the data-about which we shall have much more to say later in this paper - should have been obvious many decades ago, given that a form of $R_{\mathrm{h}}$ already appeared in the early 1900's in de Sitter's [7] first publication of his now famous solution to Einstein's equations. No doubt, the popularization of comoving coordinates by Friedmann in the 1920's [8] diverted attention away from $R_{\mathrm{h}}$, which only appears in the metric when its coefficients are written in terms of proper - rather than comoving - coordinates (see, e.g., refs. $[10,11])$. An important feature of the apparent (gravitational) horizon in cosmology is that its radius $R_{\mathrm{h}}$ is time-dependent. It is not static, like an event horizon surrounding a Schwarzschild black hole. The Universe's apparent horizon may or may not turn into a true event horizon, depending on the equation of state (i.e., pressure $p$ versus energy density $\rho$ ) in the cosmic fluid, which determines the future history of the cosmic expansion.

It is not difficult to understand why an apparent (gravitational) horizon emerges in FLRW, by simply comparing the two sets of metric coefficients written, respectively, in terms of the comoving and proper coordinates [6]. The high degree of symmetry in this metric negates any gravitational influence on the interior of a spherical shell from the rest of the Universe outside, a clear dichotomy enabled by the Birkhoff theorem and its corollary $[12,10,6]$. Not surprisingly, then, $R_{\mathrm{h}}$ is simply given by the familiar Schwarzschild form

$$
R_{\mathrm{h}}=\frac{2 G M}{c^{2}}
$$

where $M$ is the proper mass contained within a sphere of proper radius $R_{\mathrm{h}}$, i.e.,

$$
M \equiv \frac{4 \pi}{3} R_{\mathrm{h}}^{3} \frac{\rho}{c^{2}},
$$

in terms of the energy density $\rho$ in the cosmic fluid. This mass is sometimes referred to as the Misner-Sharp mass [13], since it appeared in the pioneering work of Misner and Sharp in their consideration of a general relativisitc spherical collapse, and sometimes as the MisnerSharp-Hernandez mass, for the subsequent analysis carried out by these authors [14]. As noted, however, one should not confuse the physical meaning of this $R_{\mathrm{h}}$ in the cosmological context with that of a black hole since, for these objects, the apparent horizon is static, and therefore an event horizon, though this is not yet necessarily the case for the cosmos.

This somewhat pedagogical introduction to the mass $M$ and its impact on $R_{\mathrm{h}}$ may not adequately convey the degree to which these definitions have been studied in the past. For example, Equation (1) is well constrained by the fact that only the Misner-Sharp-Hernandez designation for $M$ is consistent with the metric coefficient $g_{r r}$ in spherically-symmetric spacetimes for gravitational expansion or collapse. In spite of the fact that it can be quite difficult to 
identify the physical mass-energy for non-asymptotically flat geometries in GR [5], other possible definitions, such as the Hawking-Hayward quasilocal mass [15], are merely restatements of the Misner-Sharp-Hernandez construct.

Thus, although our definition of $R_{\mathrm{h}}$ appears to be overly simplistic, it is actually fully consistent with the already understood meaning of an apparent horizon in general relativity, including its relevance to black-hole systems [6]. More to the point of this paper, the FLRW metric is always spherically symmetric, so the general definition of an apparent horizon reduces exactly to Equation (1) $[3,5]$ and, for this reason, we shall use the descriptors 'apparent' and 'gravitational' interchangeably, or even together - as in 'the apparent (gravitational) horizon'throughout this paper.

Our principal goal here is to demonstrate why this horizon has become such an essential ingredient in the interpretation of the cosmological data, and how its measured value reveals the emergence of new physics beyond the standard model of particle physics. Its relevance to our understanding of the cosmos started to become quite evident when the optimization of free parameters in the concordance model, $\Lambda \mathrm{CDM}[16,17,18]$, revealed a very strange, unexpected coincidence - that the gravitational radius today, $R_{\mathrm{h}}\left(t_{0}\right)$, equals $c t_{0}$ to within the measurement error $[9,10,19]$. As we shall see shortly, this equality is not only surprising; it is actually highly improbable, and if we believe that there is actual physics behind its emergence, the only way to account for it is with a very special equation of state - the zero active mass condition, $\rho+3 p=0$ $[20,21]$. It is specifically this constraint that cannot accommodate a cosmological constant, replacing it instead with a dark energy density that changes with time.

Before we begin our exploration of this unusual cosmological coincidence, we should mention, as an aside, that there actually already exists another confirming indication of the importance of $R_{\mathrm{h}}$, based on how it leads to a resolution of the long-standing debate concerning the origin of cosmological redshift, i.e., whether it constitutes a new form of time dilation, or merely another instance of the better established kinematic/Doppler and gravitational effects studied in other applications of general relativity [22]. The interpretation of cosmological redshift as due to an actual expansion of space is problematic and almost certainly unphysical $[23,24,25,26,27,28,29]$. Instead, the introduction of the gravitational radius $R_{\mathrm{h}}$ proved that it is simply a product of both the Doppler and gravitational redshifts in an expanding cosmos $[22]$.

\section{The $R_{\mathrm{h}}=c t$ Hypothesis}

To understand why the equality $R_{\mathrm{h}}\left(t_{0}\right)=c t_{0}$ is unexpected and highly unlikely, let us begin by writing the spherically-symmetric FLRW metric in its standard form, using comoving coordinates $(c t, r, \theta, \phi)$, where $t$ is the cosmic time:

$$
d s^{2}=c^{2} d t^{2}-a^{2}(t)\left[d r^{2}\left(1-k r^{2}\right)^{-1}+r^{2}\left(d \theta^{2}+\sin ^{2} \theta d \phi^{2}\right)\right] .
$$

Here, $a(t)$ is the universal expansion factor, so that the 'proper' (or physical) distance is $R \equiv$ 
$a(t) r$, and the geometric constant $k$ is +1 for a closed universe, 0 for a flat, open universe, or -1 for an open universe.

Folding this metric through Einstein's field equations of general relativity, one obtains the following equations of motion describing the cosmic expansion:

$$
H^{2} \equiv\left(\frac{\dot{a}}{a}\right)^{2}=\frac{8 \pi G}{3 c^{2}} \rho-\frac{k c^{2}}{a^{2}}
$$

known as the Friedmann equation [8], and

$$
\frac{\ddot{a}}{a}=-\frac{4 \pi G}{3 c^{2}}(\rho+3 p),
$$

the so-called 'acceleration' equation. $H$ is the Hubble constant, and an overdot denotes a derivative with respect to $t$, while $\rho$ and $p$ represent, respectively, the total energy density and pressure, as noted earlier.

The Friedmann equation is actually also derivable quite easily using the Birkhoff theorem in the Newtonian limit [30, 12], which provides an intuitively satisfying meaning to the spatial curvature constant $k$. One can show that it is proportional to the sum of local positive expansion kinetic energy (related to $\mathrm{H}^{2}$ ) and local negative potential energy (represented by the term $\left.8 \pi G \rho / 3 c^{2}\right)$. The observational data seem to indicate that the Universe is spatially flat, meaning that $k=0$ [31]. Therefore, the Big Bang apparently separated positive kinetic energy and negative potential energy in equal (cancelling) portions, lending some support to the view that the Universe may have begun its expansion as a quantum fluctuation in vacuum. Together with Equations (1), (2) and (4), this result also shows that

$$
R_{\mathrm{h}}=\frac{c}{H}
$$

which is readily recognizable as the Hubble radius. The measured value of the Hubble constant today, $H_{0} \sim 70 \mathrm{~km} \mathrm{~s}^{-1} \mathrm{Mpc}^{-1}$, therefore suggests a gravitational radius for the Universe of $R_{\mathrm{h}} \sim 14$ Glyr, virtually indistinguishable from the distance $c t_{0}$ [31]. But $R_{\mathrm{h}}\left(t_{0}\right)$ could have been anything - from as little as $1 \mathrm{~cm}$ to as large as $10^{100}$ times $14 \mathrm{Glyr}$ - or more.

This range is enormous due to the flexibility of the solution to Equation (4) with $k=0$. The standard model of cosmology, known as $\Lambda$ CDM, is based on the FLRW metric with an empirically motivated choice of components in the energy density $\rho$ of the cosmic fluid. It contains (visible and dark) matter $\left(\rho_{\mathrm{m}}\right)$, radiation $\left(\rho_{\mathrm{r}}\right)$, and an unknown 'dark' energy generally assumed to be a cosmological constant $\Lambda\left(\rho_{\Lambda}\right)$, such that $\rho=\rho_{\mathrm{m}}+\rho_{\mathrm{r}}+\rho_{\Lambda}$. Each of these constituents changes with $a(t)$ in its own unique way, and conventional wisdom has it that radiation dominated at early times, while matter and, more recently, dark energy have been dominant since then. From basic physical principles we expect that $\rho_{\mathrm{m}} \sim a^{-3}$ and $\rho_{\mathrm{r}} \sim a^{-4}$, while $\rho_{\Lambda}$ would be independent 
of $a$ if dark energy were truly a cosmological constant. Therefore, defining the so-called critical density $\rho_{\mathrm{c}} \equiv 3 c^{2} H_{0}^{2} / 8 \pi G$, it is easy to see from Equation (4) that

$$
c t=R_{\mathrm{h}}\left(t_{0}\right) \int_{0}^{a} d u\left(\Omega_{\mathrm{m}} u^{-1}+\Omega_{\mathrm{r}} u^{-2}+\Omega_{\Lambda} u^{2}\right)^{-1 / 2},
$$

where $\Omega_{i} \equiv \rho_{i}\left(t_{0}\right) / \rho_{\mathrm{c}}$ for each species "i". Using the conventional normalization $a\left(t_{0}\right)=1$, we thus find that

$$
\frac{R_{\mathrm{h}}\left(t_{0}\right)}{c t_{0}}=\left\{\int_{0}^{1} d u\left(\Omega_{\mathrm{m}} u^{-1}+\Omega_{\mathrm{r}} u^{-2}+\Omega_{\Lambda} u^{2}\right)^{-1 / 2}\right\}^{-1}
$$

which is heavily dependent on the density ratios $\Omega_{\mathrm{m}}, \Omega_{\mathrm{r}}$ and $\Omega_{\Lambda}$. The problem is that these quantities are entirely empirical, presumably set by initial conditions at the time of the Big Bang, without any known theoretical underpinning. As far as we know, in the context of $\Lambda$ CDM they could have had any value.

But to be conservative, let us ignore the overall randomness in $H_{0}, \Omega_{\mathrm{m}}, \Omega_{\mathrm{r}}$ and $\Omega_{\Lambda}$, and assume, for whatever reason, that there actually does exist some unrecognized theoretical basis for the values we have measured [31, 32], i.e., $H_{0}=67.4 \pm 0.5 \mathrm{~km} \mathrm{~s}^{-1} \mathrm{Mpc}^{-1}, \Omega_{\mathrm{m}}=0.315 \pm 0.007$, $\Omega_{\mathrm{r}}=(5.48 \pm 0.001) \times 10^{-5}$ and $\Omega_{\Lambda}=1.0-\Omega_{\mathrm{m}}-\Omega_{\mathrm{r}}$. How would the ratio $R_{\mathrm{h}}(t) / c t$ as calculated from Equation (8) with these Planck optimized parameters change were we to measure it at times other than $t_{0}$ ? The answer to this question is truly puzzling because, as shown in Figure 1, even for this fixed set of parameters, $R_{\mathrm{h}}$ equals $c t$ only once in the entire history of the Universeand it must be happening right now, at time $t_{0}$, just when we happen to be looking. In other words, the fact that we infer the constraint $R_{\mathrm{h}}\left(t_{0}\right)=c t_{0}$ today is an astonishingly unlikely event. Actually, if the Universe's timeline is infinite, as one might expect for an open FLRW model with $k=0$, the probability of this happening at any particular time is essentially zero.

Equations (5) and (8) are subject to all sorts of possible accelerations and decelerations, yet the observations today are telling us that the Universe's early phase of deceleration was exactly cancelled by a subsequent acceleration, with a transition occurring roughly at the midpoint. Yet this cancellation could not have occurred - and could not ever occur - at any time other than $t_{0}$. For this reason, it is simply not sensible to accept such an eventuality as a mere coincidence. There must be some physics underlying what we see.

Of course, there could be several possible explanations for this constraint, but the simplest is simply that $R_{\mathrm{h}}$ is always equal to $c t[10,11,30]$. Then any observer, no matter when (s)he looks, would conclude that his apparent (gravitational) horizon equals the distance light could have traveled since the Big Bang. In other words, the probability of us seeing $R_{\mathrm{h}}\left(t_{0}\right)=c t_{0}$ today - or at any other time - would then be one. As we shall see shortly, the observational evidence in favour of the hypothesis $R_{\mathrm{h}}(t)=c t$ for all $t$ is now quite compelling. Later in this paper, we shall highlight some of the most striking supporting examples though, in reality, there 


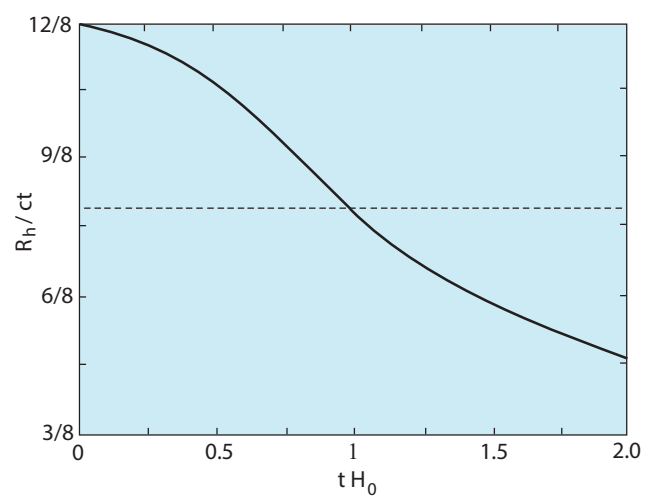

Figure 1: The ratio $R_{\mathrm{h}}(t) / c t$ as a function of $t H_{0}$, for the 291 Planck optimized parameters [31], starting at the $292 \mathrm{Big}$ Bang $\left(t H_{0}=0\right)$ and extending to twice the current age of the 293 Universe. Since the spatial curvature constant is apparently zero $294(k=0)$, the Universe is infinite, so the probability of us seeing 295 a ratio $R_{\mathrm{h}}(t) / c t=1$ today $\left(t H_{0}=1\right)$, just when we happen 296 to be looking, is essentially zero.

have now been over 24 different kinds of test completed and published in the primary literature, confirming this theory. But before we get to that discussion, let us first consider a theoretical argument that supports the actual realization of the zero active mass condition, for which the $R_{\mathrm{h}}(t)=c t$ constraint is guaranteed for all $t$.

\section{The Lapse function in FLRW Cosmologies}

The $R_{\mathrm{h}}=c t$ hypothesis has recently been validated by a theoretical study of the lapse function $g_{t t}$ in the FLRW metric. This is the coefficient multiplying the $c^{2} d t^{2}$ term in Equation (3). As is well known, the FLRW metric adopts a high degree of symmetry, including homogeneity and isotropy, making it a special member of the class of spherically-symmetric spacetimes describing gravitational collapse or expansion $[33,34,13,35]$. Unlike the other metrics in this category, however, the lapse function $g_{t t}$ in FLRW is always set equal to one - regardless of which equation of state is assumed for the stress-energy tensor $T^{\mu \nu}$.

While setting $g_{t t}=1$ is consistent with the Cosmological principle (i.e., the assumption of homogeneity and isotropy), however, there is actually no justification for supposing that the lapse function should always be coordinate-independent for any arbitrary stress-energy tensor $T^{\mu \nu}$. For a metric with $g_{t t}=1$, the observer sees no time dilation, regardless of whether or not the cosmic expansion is accelerating. This issue should be problematic at a fundamental level in GR, but is often ignored in the literature because $g_{t t}$ in FLRW can, at most, be a function only of time, not space, in order for it to be consistent with isotropy. Therefore, conventional wisdom has it that one can, if necessary, change the gauge $d t \rightarrow d t^{\prime} \equiv \sqrt{g_{t t}} d t$ to restore a coordinateindependent lapse function in FLRW, while retaining one's preferred expansion factor $a(t)$. 
Table 1: Observational Tests of the $R_{\mathrm{h}}=c t$ hypothesis and a comparison with $\Lambda$ CDM

\begin{tabular}{|c|c|c|}
\hline Test or Observational Conflict & Outcome & Reference \\
\hline Angular correlation function of the $\mathrm{CMB}$ & $R_{\mathrm{h}}=c t$ fits it very well; standard inflationary $\Lambda$ CDM misses by $\sim 6 \sigma$ & {$[38]$} \\
\hline Massive halo growth at $4 \lesssim z \lesssim 10$ & Data consistent with $R_{\mathrm{h}}=c t ; \Lambda \mathrm{CDM}$ misses by a factor $\sim 10^{4}$ & {$[39,40]$} \\
\hline Electroweak Horizon Problem & $R_{\mathrm{h}}=c t$ does not have it; $\Lambda \mathrm{CDM}$ currently has no solution & {$[41]$} \\
\hline Missing progenitors of high- $z$ quasars & In tension with $\Lambda \mathrm{CDM}$, but consistent with the timeline in $R_{\mathrm{h}}=c t$ & {$[42]$} \\
\hline Angular-diameter distance test with quasar cores & $R_{\mathrm{h}}=c t$ is favoured over $\Lambda$ CDM with BIC likelihood $81 \%$ vs $19 \%$ & {$[43,44]$} \\
\hline HII Hubble diagram & $R_{\mathrm{h}}=c t$ is favoured over $\Lambda \mathrm{CDM}$ with BIC likelihood $93 \%$ vs. $7 \%$ & {$[45,46]$} \\
\hline Alcock-Paczyński test with the BAO scale & $R_{\mathrm{h}}=c t$ is favoured over $\Lambda \mathrm{CDM}$ at a $2.6 \sigma$ confidence level & {$[47]$} \\
\hline FSRQ $\gamma$-ray luminosity function & $R_{\mathrm{h}}=c t$ is very strongly favoured over $\Lambda \mathrm{CDM}$ with $\Delta \gg 10$ & {$[48]$} \\
\hline QSO Hubble diagram + Alcock-Paczyński & $R_{\mathrm{h}}=c t$ is about 4 times more likely than $\Lambda \mathrm{CDM}$ to be correct & {$[49]$} \\
\hline Constancy of the cluster gas mass fraction & $R_{\mathrm{h}}=c t$ is favoured over $\Lambda \mathrm{CDM}$ with BIC likelihood $95 \%$ vs $5 \%$ & {$[50]$} \\
\hline Cosmic Chronometers & $R_{\mathrm{h}}=c t$ is favoured over $\Lambda$ CDM with BIC likelihood $95 \%$ vs $5 \%$ & {$[51,52]$} \\
\hline Cosmic age of old clusters & $\Lambda \mathrm{CDM}$ cannot accommodate high- $z$ clusters, but $R_{\mathrm{h}}=c t$ can & {$[53]$} \\
\hline High- $z$ quasars & The evolution timeline fits within $R_{\mathrm{h}}=c t$, but not $\Lambda$ CDM & {$[54,55,56]$} \\
\hline The AGN Hubble diagram & $R_{\mathrm{h}}=c t$ is favoured over $\Lambda$ CDM with BIC likelihood $96 \%$ vs $4 \%$ & {$[57]$} \\
\hline Age vs. redshift of old passive galaxies & $R_{\mathrm{h}}=c t$ favoured over $\Lambda$ CDM with BIC likelihood $80 \%$ vs $20 \%$ & {$[58]$} \\
\hline Type Ic superluminous supernovae & $R_{\mathrm{h}}=c t$ is favoured over $\Lambda$ CDM with BIC likelihood $80 \%$ vs $20 \%$ & {$[59]$} \\
\hline The SNLS Type Ia SNe & $R_{\mathrm{h}}=c t$ is favoured over $\Lambda$ CDM with BIC likelihood $90 \%$ vs $10 \%$ & {$[60]$} \\
\hline Angular size of galaxy clusters & $R_{\mathrm{h}}=c t$ is favoured over $\Lambda$ CDM with BIC likelihood $86 \%$ vs $14 \%$ & {$[61]$} \\
\hline Strong gravitational lensing galaxies & Both models fit the data very well due to the bulge-halo 'conspiracy' & {$[62,63]$} \\
\hline Time delay lenses & $R_{\mathrm{h}}=c t$ is favoured over $\Lambda \mathrm{CDM}$ with BIC likelihood $80 \%$ vs $20 \%$ & {$[64]$} \\
\hline High- $z$ galaxies & The evolution timeline fits within $R_{\mathrm{h}}=c t$, but not $\Lambda \mathrm{CDM}$ & {$[65]$} \\
\hline GRBs + star formation rate & $R_{\mathrm{h}}=c t$ is favoured over $\Lambda \mathrm{CDM}$ with AIC likelihood $70 \%$ vs $30 \%$ & {$[66]$} \\
\hline High- $z$ quasar Hubble diagram & $R_{\mathrm{h}}=c t$ is favoured over $\Lambda \mathrm{CDM}$ with BIC likelihood $85 \%$ vs $15 \%$ & {$[67]$} \\
\hline GRB Hubble diagram & $R_{\mathrm{h}}=c t$ is favoured over $\Lambda$ CDM with BIC likelihood $96 \%$ vs $4 \%$ & {$[68]$} \\
\hline
\end{tabular}

But this complacency has been challenged recently with an application of the Local Flatness Theorem (see, e.g., refs. [36]) to examine whether 'creating' such a frame of reference is in fact consistent with the GR mandated existence of a local free-falling frame against which one can always measure the spacetime curvature and concomitant time dilation in the given metric [37]. This is a somewhat subtle point, but fundamentally rooted in the equivalence principle. The Local Flatness Theorem shows that the conflict between a unitary value of the lapse function and an arbitrary choice of $a(t)$ cannot be removed with a simple change of gauge which, in relativity, is actually a transformation of the coordinates, i.e., a change in reference frames [20].

The Local Flatness Theorem compels [37] the FLRW lapse function to satisfy the following constraint:

$$
\int^{c t} g_{t t}\left(t^{\prime}\right) d\left(c t^{\prime}\right)=c g_{t t}(t) \frac{a}{\dot{a}}
$$

Therefore, the choice of lapse function $g_{t t}(t)=1$ is consistent only with the expansion factor

$$
a(t) \equiv\left(\frac{t}{t_{0}}\right)
$$

normalized in conventional form for a spatially flat metric with $a\left(t_{0}\right)=1$. (There is, of course, also the trivial solution $a=$ constant, for which the spacetime curvature is identically zero, and the coordinates of the FLRW metric in Equation 3 are then those in Minkowski space.) 
A quick inspection of the acceleration Equation (5) therefore shows that there is only one equation of state consistent with the choice of lapse function $g_{t t}=1$ (other than the inconsequential $a=$ constant case, for which $\rho=p=0$ ), and this is the so-called zero active mass condition, $\rho+3 p=0$, which produces a constant universal expansion rate. But this is not the empty Milne universe, in which $\rho=p=0$ and the linear expansion is driven by spatial curvature with $k=-1$. It is trivial to see from Equation (4) that a flat FLRW Universe must always have a Hubble constant $H(t)=1 / t$ and an apparent (gravitational) radius $R_{\mathrm{h}}(t)=c t$.

What appeared to be an unlikely coincidence in Figure 1 turns out to be well justified theoretically. One may wonder, then, how the standard model of cosmology, which has always ignored the role played by $R_{\mathrm{h}}$, successfully accounts for many of the observations. As we shall see below, $\Lambda \mathrm{CDM}$ has served us well with gross interpretations of the data, but not so well once the measurement precision started to improve. Today, the tension between its predictions and the observations is too large to ignore and the disparity between standard theory and experiment in several areas is quite glaring. In subsequent sections of this paper, we shall also highlight the contrasting success of the $R_{\mathrm{h}}=c t$ hypothesis in fixing (or removing) all of these emerging problems.

\section{Observational Tests of the $R_{\mathrm{h}}=c t$ Hypothesis}

An initial assessment of the preceeding discussion might seem to be at odds with the standard model's success in accounting for a broad range of cosmological data. After all, $\Lambda$ CDM contains dark energy in the form of a cosmological constant, $\Lambda$, with pressure $p_{\Lambda}=-\rho_{\Lambda}$ and a total equation of state based on individual variations of the energy density for each species. Therefore, $p=p_{\mathrm{m}}+p_{\mathrm{r}}+p_{\Lambda}$ in this model is unavoidably an evolving function of $\rho=\rho_{\mathrm{m}}+\rho_{\mathrm{r}}+\rho_{\Lambda}$.

It is thus crucial, not only to gauge how the $R_{\mathrm{h}}=c t$ equality fares on average over a Hubble time but, to simply impose the zero active mass condition on $\Lambda$ CDM for all $t$ and examine whether it mitigates or eliminates the tension growing between theory and observation. This effort has been underway for over a decade now, including over 24 different kinds of measurement, at low and high redshifts, using integrated times and distances, and also the differential rate of expansion, $H(z)$. A summary of the completed tests is provided in Table 1, along with references to the literature where the results have been published. It is quite remarkable that-perhaps contrary to expectation - the equation of state $\rho+3 p=0$ reduces or eliminates any disparity between the data and model predictions in every case studied thus far.

The outcomes shown in this table are based on model selection tools designed to 'choose' which model (or models) are preferred by the data. The Akaike Information Criterion (AIC [69]), the Kullback Information Criterion (KIC [70]), and the Bayes Information Criterion (BIC [71]) are used often for this purpose. In terms of the maximum value of the likelihood function $\mathcal{L}$ and the number $n$ of free parameters in the model, the difference $\Delta_{\mathrm{IC}}=\mathrm{IC}_{2}-\mathrm{IC}_{1}$ determines the extent to which model $\mathcal{M}_{1}$ is preferred over model $\mathcal{M}_{2}$, with $\mathrm{IC}_{\alpha}=-2 \ln \mathcal{L}_{\alpha}+2 n_{\alpha}$, and 


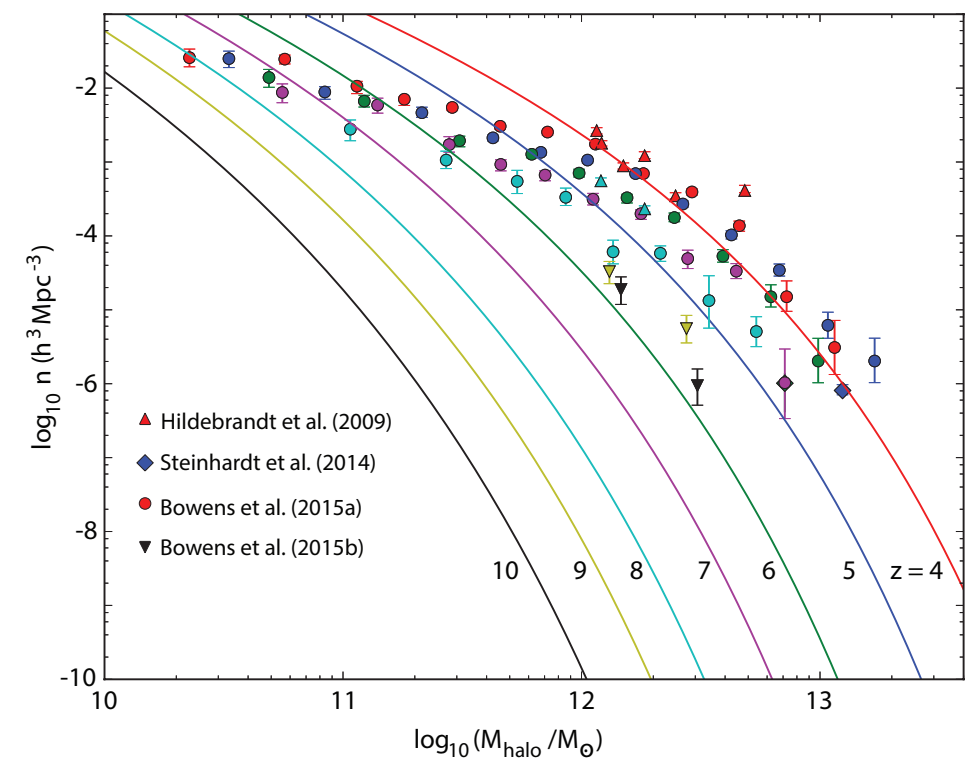

Figure 2: Halo mass function inferred from galaxy surveys, as a function of mass and redshift: $z=4$ (red), 5 (blue), 6 (green), 7 (magenta), 8 (cyan), 9 (yellow), and 10 (black). Solid curves represent the theoretical halo number density predicted by $\Lambda \mathrm{CDM}$ in this same redshift range, based on the estimates of ref. [72] and calculated with the HMFCalc code of ref. [73]. (Adapted from ref. [39])

IC is either AIC, KIC, or BIC, as the case may be [51]. The outcome $\Delta_{\text {IC }}$ is considered to represent 'positive' evidence that model 1 is preferred over model 2 when $\Delta_{\mathrm{IC}}>2$; 'moderate' when $2<\Delta_{\text {IC }}<6$; and very strong when $\Delta_{\text {IC }}>10$. One may also use $\Delta_{\text {IC }}$ to estimate the relative probability (or percentage likelihood) that model 1 is statistically preferred over model 2 , according to the formulation

$$
P\left(\mathcal{M}_{1}\right)=\frac{1}{1+\exp \left(-\Delta_{\mathrm{IC}} / 2\right)},
$$

with $P\left(\mathcal{M}_{2}\right)=1-P\left(\mathcal{M}_{1}\right)$, in a head-to-head comparison between two models.

Table 1 shows that all of the comparative tests concluded thus far consistently favour $R_{\mathrm{h}}=c t$ over basic $\Lambda \mathrm{CDM}$ without the zero active mass equation of state. The preference is sometimes moderate, and often strong. For illustration we here highlight two of the most prominent examples where continued support for basic, inflationary $\Lambda$ CDM over $R_{\mathrm{h}}=c t$ appears to be untenable. These are (1) the inferred halo distribution as a function of mass and redshift in the redshift range $4 \lesssim z \lesssim 10$, which disagrees with structure formation in $\Lambda$ CDM by as much as four orders of magnitude; and (2) the angular correlation function of the CMB, which shows strong evidence of a minimum cutoff in the power spectrum, conflicting with the basic 


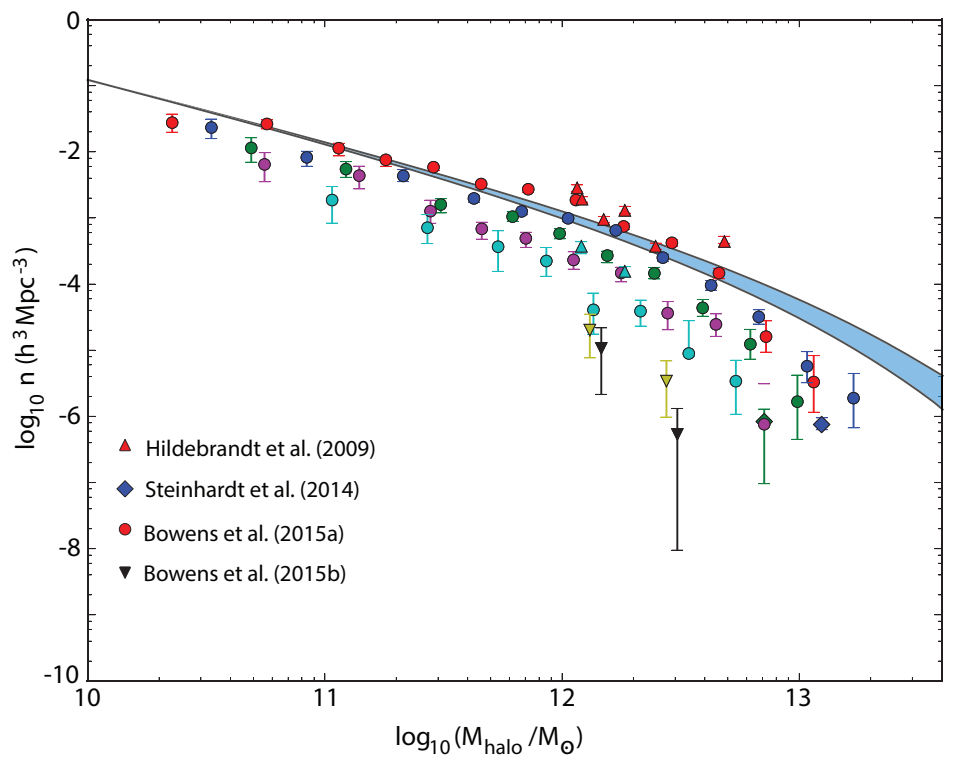

Figure 3: Same as figure 2, except that the data have been recalibrated for the $R_{\mathrm{h}}=c t$ universe using the ratio of differential comoving volumes [40]. The solid curves represent the halo mass function for $R_{\mathrm{h}}=c t$, using a normalization based on the optimized fit to recently published redshift space distortion measurements of the cosmological growth rate [74]. The halo mass function in $R_{\mathrm{h}}=c t$ is essentially independent of redshift in the range $4 \lesssim z \lesssim 10$. (Adapted from ref. [40])

inflationary paradigm.

The halo mass data are shown in figure 2, together with seven theoretical curves calculated for $4 \lesssim z \lesssim 10$, using the halo mass function estimates of ref. [72], and the HMFCalc code developed in ref. [73]. The $\Lambda$ CDM parameters are assumed to have their Planck values (see $\S$ II above and ref. [31]). The disparity between the predicted and inferred distributions is quite pronounced. The surprisingly early appearance of massive galaxies significantly challenges the standard model, and the halo mass function at $z \gtrsim 4$ is grossly inconsistent with the predictions of $\Lambda$ CDM, a situation termed "The Impossibly Early Galaxy Problem" by workers in the field [39]. The most significant tension is caused by the lack of anticipated strong evolution in redshift and the absence of a steepening of the distribution with increasing mass.

The unexpected nature of the halo mass function has also been characterized as a problem with the theory of galaxy formation at high $z$, but this outcome is not new. The observed highredshift quasars and galaxies would have formed much too quickly compared to the timeline in the standard model $[54,65]$. To account for the emergence of $\sim 10^{9} M_{\odot}$ black holes earlier than $z \sim 6-7$ in $\Lambda$ CDM, one must assume that black holes started growing from anomalously 


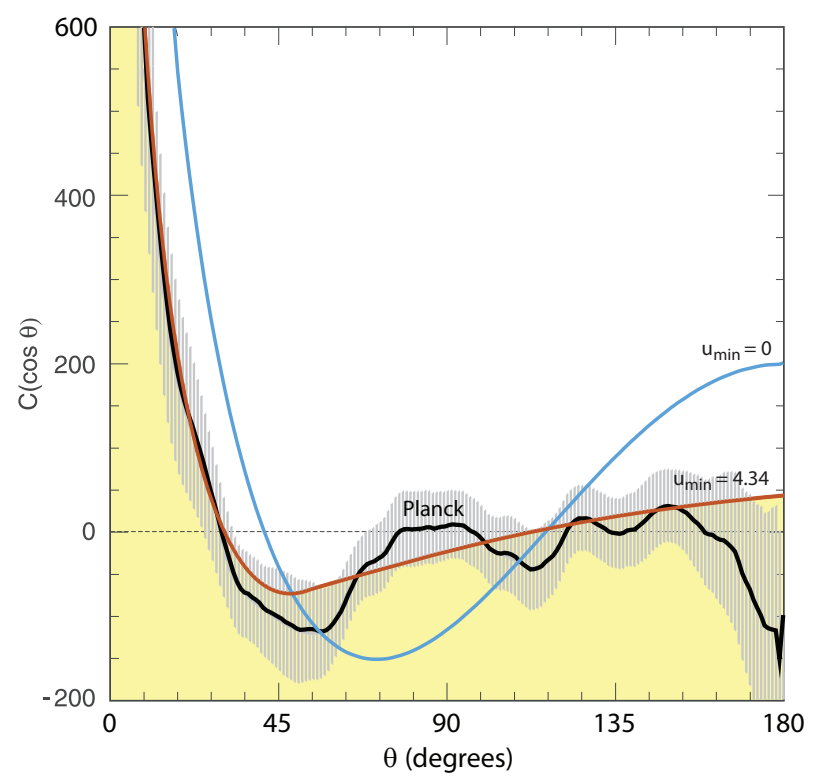

Figure 4: Angular correlation function measured with Planck (dark solid curve) [75], including $1 \sigma$ errors (grey), compared with (blue) the conventional inflationary $\Lambda$ CDM prediction, and (red) truncated inflation, or a non-inflationary cosmology [38]. The parameter $u_{\min }$ is proportional to the minimum wavenumber in the power spectrum. A value of 0 , predicted by inflationary $\Lambda \mathrm{CDM}$, is ruled out at a level of significance greater than $6 \sigma$. In $R_{\mathrm{h}}=c t$, the measured value of $u_{\text {min }}$ roughly corresponds to the Planck scale, where the quantum fluctuations enter the classical Universe [76].

large seeds $\left(M>10^{5} M_{\odot}\right)$, or evolved at super-Eddington rates, neither of which has ever been justified on astrophysical grounds. By comparison, the timeline in $R_{\mathrm{h}}=c t$ would have allowed these high-redshift objects to form following well understood astrophysical principles $[54,65,55]$.

Insofar as the halo masses are concerned, linear perturbation theory in $R_{\mathrm{h}}=c t$ predicts the distribution shown in figure 3 [40]. The dependence on mass and redshift is far more compatible with the data than the corresponding prediction by $\Lambda$ CDM. There is still work to be done, however, because the anticipated distribution still deviates from the observations by some unidentified systematic effect at the highest masses.

A comparably serious problem has emerged in the angular correlation function of the CMB, starting with the Wilkinson Microwave Anisotropy Probe (WMAP) [16, 17] and resoundingly confirmed by Planck [75]. This is but one of several anomalies seen on very large scales, though the lack of any significant correlation seen at angles $\gtrsim 60^{\circ}$ is easily the most glaring one. The fact that it is inconsistent with the basic inflationary concept has understandably initiated vigorous debate about whether it is real, or due to some unknown observational systematic effect. It could, e.g., be due to an improper foreground subtraction [77], but after three independent missions 
have all confirmed this problem (COBE preceded WMAP and Planck), it is difficult to accept that subtleties in the foreground subtraction have yet to be resolved following three decades of observation. The lack of large-angle correlations could also be due to cosmic variance [77, 78], but the probability of this actually happening is typically $\lesssim 0.24 \%$, showing an inconsistency with the inflationary paradigm at better than $3 \sigma[79,80,81]$.

This disparity is quite serious because - whereas anisotropies measured at angles $\lesssim 1^{\circ}$ are mostly due to local astrophysical processes (such as acoustic wave propagation) - the large-scale fluctuations reflect the physics of dynamical expansion, i.e., the cosmological model itself. To study this problem in greater detail, a re-analysis of the large-scale anisotropies using a recent Planck release [75] therefore took a different approach [38]. Instead of following convention established by the inflationary picture, this assumed that quantum fluctuations generated in the early Universe had a well-defined power spectrum $P(k)$ with a wavenumber cutoff $k_{\min } \neq 0$. Basic inflationary models do not have such a physical scale, so if the CMB data rule out a zero $k_{\min }$, this would be compelling evidence against the inflationary solution to the horizon problem and, by association, the early phase of decelerated expansion predicted by $\Lambda$ CDM.

Figure 4 shows the CMB angular correlation function measured in this recent analysis (solid black), together with the $\Lambda$ CDM prediction (blue) and the optimized fit (red) using a non-zero $k_{\min }$. The parameter $u_{\min }$ is proportional to $k_{\min }$ and is defined as follows: $u_{\min } \equiv k_{\min } c \Delta \tau_{\text {dec }}$, where $c \Delta \tau_{\text {dec }}$ is the comoving radius of the last scattering surface (at 'decoupling') in terms of the conformal time difference between $t_{0}$ and $t_{\mathrm{dec}}$. The lack of adequate confirmation by the data of the standard model prediction is difficult to hide. The best-fit (red) curve corresponds to an optimized value $u_{\min }=4.34 \pm 0.50$, implying a maximum fluctuation size $\theta_{\max } \approx 83^{\circ}$ in the plane of the sky. In $\Lambda \mathrm{CDM}$, decoupling is thought to have occurred at $z_{\mathrm{dec}}=1080$, so this measurement of $u_{\min }$ corresponds to a maximum fluctuation wavelength $\lambda_{\max } \sim 20 \mathrm{Mpc}$ at that redshift.

There are several reasons why the red curve is strongly preferred over the standard model's prediction, including the fact that it not only accounts for the lack of large-angle fluctuations, but actually fits the measurements to within $1 \sigma$ at all angles; and it correctly accounts for the amplitude of the minimum of the angular correlation function and the angle at which it occurs. Most importantly, these results show that a non-zero cutoff in $P(k)$ is favoured over $k_{\min }=0$ at a confidence level exceeding $\sim 8 \sigma$.

But while a $k>k_{\text {min }}$ constraint on $P(k)$ is inconsistent with slow-roll inflationary cosmology, it is actually expected with the $R_{\mathrm{h}}=c t$ hypothesis [10, 30, 76]. A viable scenario with this picture has the fluctuations emerging at or near the Planck scale, which equals the apparent (gravitational) horizon at the Planck time. The non-zero value of $k_{\min }$ therefore corresponds to the size of this horizon at $t_{\mathrm{dec}}$, since only fluctuations with a size $\lesssim \lambda_{\max } \sim 2 \pi R_{\mathrm{h}}$ would have grown continuously towards the last scattering surface. In the next section, we shall discuss the consequences of this work on the viability of the inflationary paradigm in more detail, together 


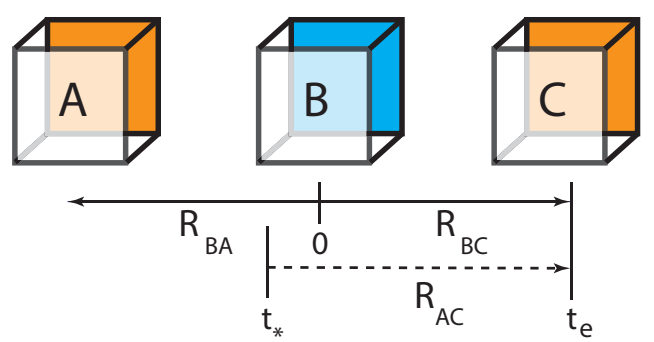

Figure 5: Observer $\mathrm{B}$ receives light signals from two regions (A and $\mathrm{C}$ ) in the CMB. Light was emitted by these sources at cosmic time $t_{e}$, a proper distance $R_{B A}\left(t_{e}\right)=R_{B C}\left(t_{e}\right)$ from the observer. Patch (A) emitted a light signal at the earlier time $t_{*}$ that reached (C), a proper distance $R_{A C}\left(t_{e}\right)$ away, at time $t_{e}$. (Adapted from ref. [82])

with another 'horizon' problem now emerging, based on the vacuum expectation value of the Higgs field.

\section{New Physics}

As we have already hinted earlier, and will demonstrate more formally in $\S \mathrm{V}$.B below, a universe with $R_{\mathrm{h}}=$ ct cannot be driven by a blended equation of state for matter, radiation and a cosmological constant. This exclusion has several significant consequences, including (1) that it implies a need to extend particle physics beyond the standard model, and (2) that it provides a simple, elegant solution to the so-called horizon problem, arising from the uniformity of both the CMB temperature across the sky and the expectation value of the Higgs field. We begin our discussion with the latter, which has plagued theoretical cosmology for over half a century.

\subsection{The Temperature and Electroweak Horizon Problems}

The CMB temperature horizon problem is a major shortcoming of $\Lambda \mathrm{CDM}$ because - without some anomalously accelerated expansion at early times - the Universe would have required highly improbable, specialized initial conditions. The microwave background radiation has the same temperature everywhere, except for tiny, random fluctuations of one part in 100,000. We infer that opposite sides of the sky (patches $\mathrm{A}$ and $\mathrm{C}$ in figure 5) lie beyond each other's horizon (which is actually not completely correct, but this is not the principal difficulty), yet their temperatures today are identical.

The temperature horizon problem in $\Lambda \mathrm{CDM}$ is best understood by studying the diagram in figure 6. For an observer at (C), the curves labeled $R_{\gamma 0}$ and $R_{\gamma \mathrm{e}}$ show the null geodesics reaching him/her at times $t_{0}$ and $t_{\mathrm{e}}$, respectively. Correspondingly, the curves labeled $R_{\mathrm{AC}}$ and $R_{\mathrm{BC}}$ give the proper distances between (A) and (C), and between (B) and (C), as functions of time. So light emitted in patch (B) at spacetime point $\delta$ would reach $C$ today (at time $t_{0}$ ), while light emitted in patch (A) at $\alpha$ would have reached (C) at time $t_{e}$. Light emitted by one of them 


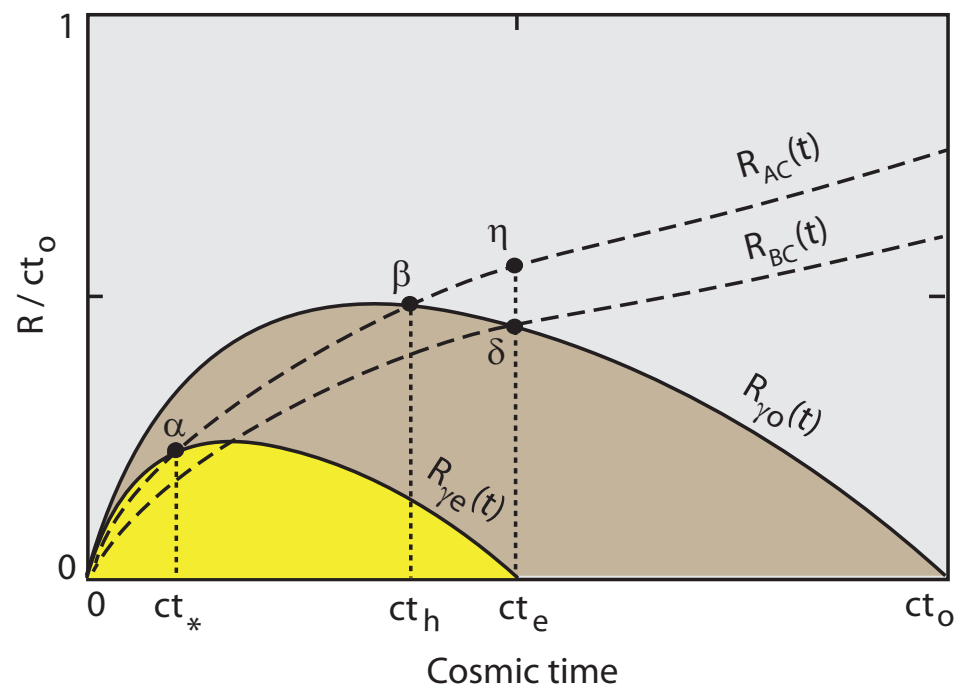

Figure 6: Proper distance $R_{\gamma 0}\left(R_{\gamma \mathrm{e}}\right)$ of light approaching an observer in patch (C) at time $t_{0}$ $\left(t_{\mathrm{e}}\right)$ as a function of $t$, and the corresponding proper distances $\left(R_{\mathrm{AC}}\right.$ and $\left.R_{\mathrm{BC}}\right)$ of $(\mathrm{A})$ and $(\mathrm{B})$ relative to $(\mathrm{C})$, all calculated in the $\Lambda \mathrm{CDM}$ cosmology. (Adapted from ref. [82])

(say patch $\mathrm{A}$ ), at $t_{*}<t_{\mathrm{e}}$, could have reached (C) prior to the emission of the CMB at $t_{\mathrm{e}}$, but in $\Lambda \mathrm{CDM}$ there is no value of $t_{*} \geq 0$ for which $R_{\mathrm{AC}}\left(t_{\mathrm{e}}\right)=2 R_{\mathrm{BC}}\left(t_{\mathrm{e}}\right)$, with an $R_{\mathrm{BC}}\left(t_{0}\right)$ bigger than the proper size of our visible Universe [82]. Therefore, an observer at (B) would not able to see a uniform temperature today.

The root of this problem is simply that the early Universe in $\Lambda$ CDM decelerated following the Big Bang, preventing patches $(\mathrm{A})$ and $(\mathrm{C})$ from receeding sufficiently from (B) prior to producing the CMB at $t_{\mathrm{e}}$. But this problem is completely absent in $R_{\mathrm{h}}=c t$ because $R_{A C}$ was always equal to $2 R_{B C}$ (see figure 7 ). So no matter the time $t_{\mathrm{e}}$ at which decoupling took place, one can always find a $t_{*}>0$ at which the emission of a signal by either patch could have reached and equilibrated the physical conditions in the other. In other words, $R_{\mathrm{h}}=c t$ never had a horizon problem to begin with.

This great simplification and avoidance of a fundamental inconsistency in the expansion dynamics is a major strength of the $R_{\mathrm{h}}=c t$ hypothesis, more so, because $\Lambda$ CDM without the zero active mass equation of state is facing a daunting task to overcome its horizon problems. The most likely solution comes from particle physics, which suggests that several phase transitions might have occurred, including an inflationary event from the separation of the strong and electroweak forces in grand unified theories (GUTs) [83, 84]. The accelerated expansion at $t \sim 10^{-36}-10^{-33}$ seconds might have greatly enlarged the proper distances $R_{A C}\left(t_{\mathrm{e}}\right)$ and $R_{B C}\left(t_{\mathrm{e}}\right)$ in figure 6 beyond our causal horizon, allowing us to see a uniform CMB temperature everywhere. Even after three decades of development, however, we still do not have a complete, self-consistent 


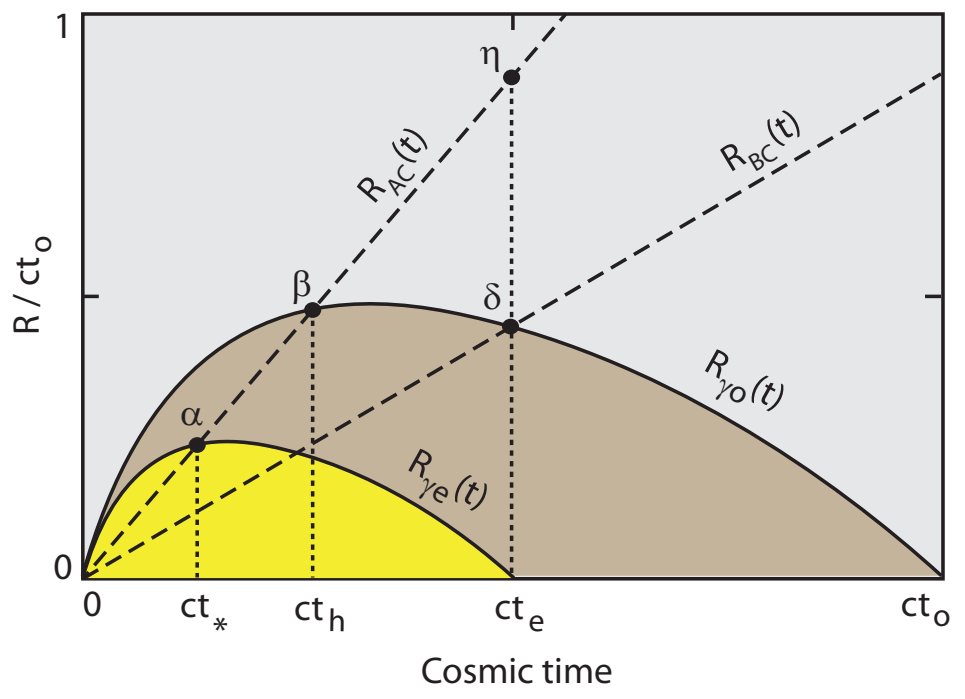

Figure 7: Same as figure 6, except now for a universe with $R_{\mathrm{h}}=c t$, i.e., with the zero active mass equation of state. Note that, unlike the situation in figure 6 , this time $R_{\mathrm{AC}}\left(t_{e}\right)=2 R_{\mathrm{BC}}\left(t_{e}\right)$. (Adapted from ref. [82])

theory of inflation $[85,86]$. We do not know the inflaton potential, nor do we have the transPlancking physics necessary to describe how the inflaton field could have emerged into the classical Universe from its remote early beginning inaccessible to modern quantum mechanics.

Now that the Higgs particle has been discovered, the situation for $\Lambda$ CDM is much worse, because the Universe probably passed through another (electroweak) phase transition at $T=$ $159.5 \pm 1.5 \mathrm{GeV}$ - about $\sim 10^{-11}$ seconds following the Big Bang. This 'turning on' of the Higgs field gave mass to the fermions and separated the weak and electric forces. The problem is that the vacuum expectation value of the Higgs field is expected to be uniform only within a region that was causally connected at the time of the phase transition. In $\Lambda \mathrm{CDM}$, that region would have expanded to about $40 \mathrm{Mpc}$ today [41]. The proper size of our visible Universe, however, is approximately 2, $212 \mathrm{Mpc}$, roughly 50 times larger, yet no evidence of variable particle properties, such as lepton mass, has ever been seen. Unlike the GUT transition that might have produced inflation to fix the CMB temperature horizon problem, there is no known solution to this equally troubling Higgs horizon inconsistency. The Higgs horizon problem is actually worse than the CMB temperature inconsistency because Higgs physics is now understood better than GUTs.

\subsection{Dynamical Dark Energy}

With the $R_{\mathrm{h}}=c t$ hypothesis, the cosmic fluid must maintain a fixed (total) equation-of-state $w \equiv p / \rho=-1 / 3$. Thus, given the dependence of $\rho_{\mathrm{m}}$ and $\rho_{\mathrm{r}}$ on the expansion factor $a(t)$ (see $\S$ II), it is not possible to do this with a cosmological constant, whose pressure is fixed at $p_{\Lambda}=-\rho_{\Lambda}$. 


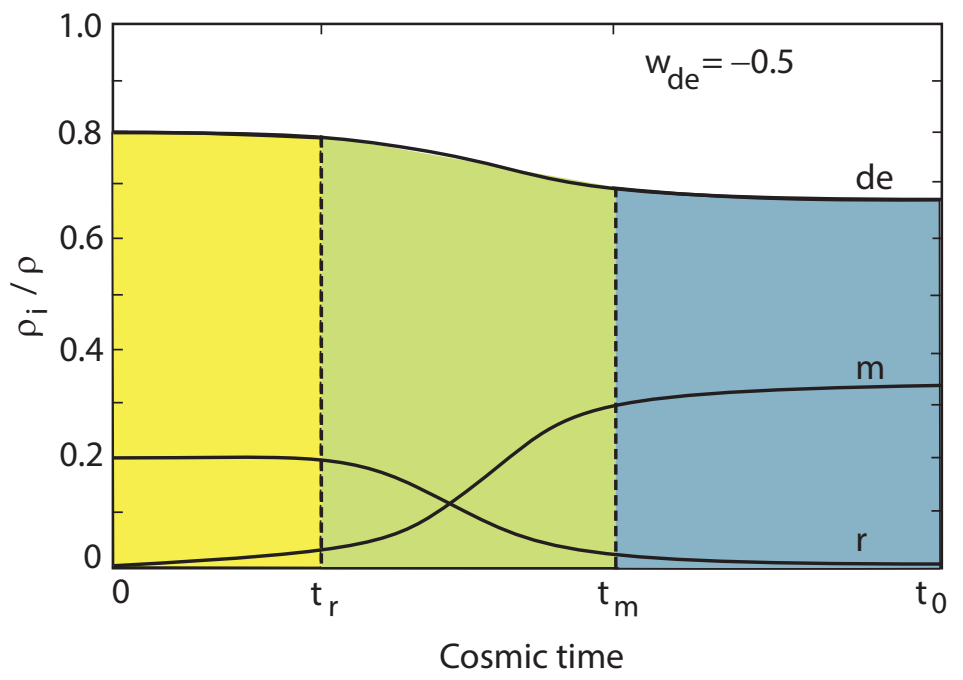

Figure 8: Schematic diagram of the possible evolution in time of various energy densities $\rho_{i}$ in $R_{\mathrm{h}}=$ ct: dark energy $(\mathrm{de})$, radiation $(\mathrm{r})$ and matter $(\mathrm{m})$. The empirical evidence today suggests that $w_{\mathrm{de}}=-0.5$, so $\rho_{\mathrm{r}} / \rho=0.2$ and $\rho_{\mathrm{de}} / \rho=0.8$ at $z \gg 1$, while $\rho_{\mathrm{m}} / \rho=1 / 3$ and $\rho_{\text {de }} / \rho=2 / 3$ for $z \sim 0$. The temperature and overall density increase back to the Big Bang, so radiation dominates over matter early on (i.e., in the region $t<t_{\mathrm{r}}$ ), while matter dominates over radiation at late times (i.e., for $t>t_{\mathrm{m}}$ ).

As we shall see, the dark energy density must evolve along with the other particle species, so it must be dynamic - perhaps a new particle (or particles) in extensions to the standard model. In this section, we estimate how $\rho_{\text {de }}$ and $p_{\text {de }}$ must have changed over a Hubble time in order to comply with the zero active mass condition.

Putting $\rho=\rho_{\mathrm{r}}+\rho_{\mathrm{m}}+\rho_{\mathrm{de}}$, and $p=-\rho / 3=w_{\mathrm{de}} \rho_{\mathrm{de}}+\rho_{\mathrm{r}} / 3$, we immediately see that

$$
\rho_{\mathrm{r}}=-3 w_{\mathrm{de}} \rho_{\mathrm{de}}-\rho,
$$

under the assumption that $p_{\mathrm{r}}=+\rho_{\mathrm{r}} / 3$ and $p_{\mathrm{m}} \approx 0$. From the Friedmann Equation (4) with spatial flatness $(k=0)$, we also recognize that, throughout cosmic history, the overall density has evolved according to

$$
\rho(t)=\rho_{\mathrm{c}} a(t)^{-2},
$$

where $\rho_{\mathrm{c}}$ is the previously defined critical density.

Equation (12) is valid at any epoch. At low redshifts, however, the empirical evidence tells us that the CMB temperature $\left(T_{0} \approx 2.728 \mathrm{~K}\right)$ translates into a negligibly small normalized radiation energy density, $\Omega_{\mathrm{r}} \sim 5 \times 10^{-5}$, compared to matter and dark energy. It is easy to see from the definitions of $\rho$ and $p$ that $w_{\text {de }}$ must therefore be $\sim-1 / 2$ in order to reflect the partitioning of $\rho_{\mathrm{m}}$ and $\rho_{\mathrm{de}}$ that we observe in the local Universe. For convenience, we shall 
simply put $w_{\mathrm{de}}=-1 / 2$. In that case,

$$
\Omega_{\mathrm{de}} \approx-\frac{1}{3 w_{\mathrm{de}}} \approx \frac{2}{3}
$$

and

$$
\Omega_{\mathrm{m}} \approx \frac{1+3 w_{\mathrm{de}}}{3 w_{\mathrm{de}}} \approx \frac{1}{3}
$$

in which, of course, $\Omega_{\mathrm{m}}$ is the sum of both baryonic and dark matter, i.e., $\Omega_{\mathrm{m}}=\Omega_{\mathrm{b}}+\Omega_{\mathrm{d}}$ [87].

As noted earlier, we expect that radiation becomes dominant over matter at large redshifts. Radiation on its own, however, cannot produce a zero active mass equation of state. Thus, to comply with the $R_{\mathrm{h}}=c t$ constraint, dark energy must be present along with radiation in the early Universe. We thus put $\rho \approx \rho_{\mathrm{r}}+\rho_{\mathrm{de}}$. In that case, we see that

$$
\rho_{\mathrm{de}} \approx \frac{2}{1-3 w_{\mathrm{de}}} \rho_{\mathrm{c}}(1+z)^{2} \quad(z \gg 1)
$$

and

$$
\rho_{\mathrm{r}} \approx \frac{3 w_{\mathrm{de}}+1}{3 w_{\mathrm{de}}-1} \rho_{\mathrm{c}}(1+z)^{2} \quad(z \gg 1)
$$

and if we also assume that $w_{\mathrm{de}}=-1 / 2$ remains constant throughout cosmic evolution, then $\rho_{\mathrm{de}}=0.8 \rho$ and $\rho_{\mathrm{r}}=0.2 \rho$ at high redshifts. These trends are shown schematically in figure 8 .

This simple argument suggests that the zero active mass equation of state $\rho+3 p=0$ requires a gradual transition of the equilibrium partitioning of the various constituents. We infer that, as a fraction of the total energy density, dark energy decreases fractionally from $\rho_{\mathrm{de}} / \rho=0.8$ when $z \gg 1$, to $\rho_{\mathrm{de}} / \rho=2 / 3$ at the present. During this evolution, radiation gradually dilutes, from $\rho_{\mathrm{r}} / \rho=0.2$ in the early Universe, to an insignificant fraction at the present time, and the fractional representation of matter increases from an insignificant amount at $z \gg 1$ to $\rho_{\mathrm{m}} / \rho=1 / 3$ at $z \rightarrow 0$.

These couplings and interactions provide us with some clues into the nature of dark energy in $R_{\mathrm{h}}=c t$. As we see in figure 8 , the radiation energy density could not have diluted according to $(1+z)^{4}$ at high redshifts, so it may have coupled to dark energy. Its designation as 'dark' would then depend on redshift, since it may have coupled to the electromagnetic (or even the electroweak) field at very high densities and temperatures. In the transition region $t_{\mathrm{r}} \lesssim t \lesssim t_{\mathrm{m}}$, the gradual decrease in the ratio $\rho_{\mathrm{de}} / \rho$ and increase in $\rho_{\mathrm{m}} / \rho$ may signal a partial decay of dark energy into standard model particles. If the dark energy fraction is plateauing now, perhaps this is also an indication that there were multiple dark energy particles, one or more of which completely decayed prior to cosmic time $t_{\mathrm{m}}$, while the rest have remained more stable. 


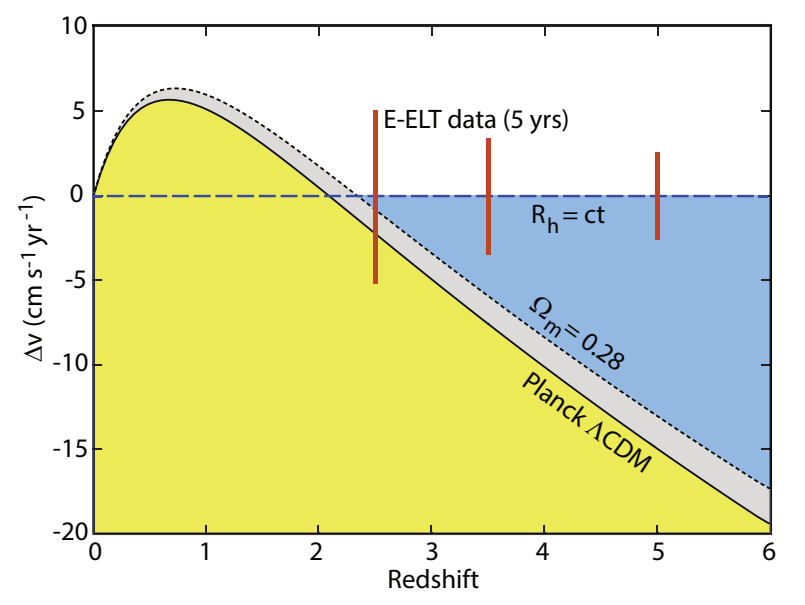

Figure 9: Predicted velocity shift $\Delta v$ underlying the redshift drift for three cosmological models [88]: (solid black) Planck $\Lambda \mathrm{CDM}\left(k=0, \Omega_{m}=0.315 H_{0}=67.4 \mathrm{~km} \mathrm{~s}^{-1} \mathrm{Mpc}^{-1}\right)$ [31]; (black dashed) a slight variation of $\Lambda \mathrm{CDM}$ with $\Omega_{m}=0.28$; and (blue long dash) the $R_{\mathrm{h}}=c t$ hypothesis, for which $\Delta v=d z / d t_{0}=0$ at all redshifts. The red bars indicate the expected $1 \sigma$ errors at $z=2.5,3.5$, and 5.0 with the ELT-HIRES after 5 years of monitoring. (Adapted from ref. [89])

\section{Conclusions and Future Prospects}

In this paper, we have argued for the paramount importance of the apparent (gravitational) horizon in cosmology, whose direct influence on our inerpretation of the data suggests a zero active mass equation of state $\rho+3 p=0$ in the cosmic fluid. This conclusion is based, at least initially, on the most likely explanation for the highly improbable coincidence of us seeing $R_{\mathrm{h}}\left(t_{0}\right)=c t_{0}$ today. The solution would simply be that this equality is actually maintained at all times. More recent theoretical work has shown that this outcome may ultimately have a fundamental basis in general relativity, which limits the applicability of the FLRW metric to stress-energy tensors that allow only a non-accelerated expansion of the Universe. As we now understand, a cosmos with a constant expansion rate necessarily satisfies the $R_{\mathrm{h}}=c t$ constraint on its apparent (gravitational) horizon.

But how should one understand the dynamics of dark energy, whose own equation of state is just right to ensure the zero active mass condition overall? Given the panoply of clues from nature, it seems reasonable to assume that the single most important feature of the Big Bang was the magnitude of the separated (positive) expansion and (negative) potential energies. This initial condition directly affects the Hubble constant, and all observational signatures that stem from it, including the angular-diameter and luminosity distances, and age versus redshift relation, among many others.

If we further maintain that the Universe ought to be homogeneous and isotropic, and 
simultaneously adopt Weyl's postulate (which essentially says that no two worldlines may ever cross), general relativity leaves us with only one kind of orderly expansion - that dictated by the FLRW spacetime, at a rate determined by $H(z)$, whose primacy results from the magnitude of the initially separated energies. In this view, it is therefore not the behaviour of the various constituents that determines the expansion profile, as one finds in $\Lambda$ CDM but, rather, the expansion rate is mandated by the Hubble constant and the properties of FLRW encoded within GR. It is the partitioning of the various constituents that must follow suit. A useful analogy here is the chemistry imposed on various interacting elements inside a piston, whose externally driven evolution in pressure and volume adjusts the equilibrium densities of the interior components. In other words, the dynamical behaviour of all the constituents in the cosmic fluid, including dark energy, is driven by the orderly FLRW expansion, with a rate based on the initial magnitude of the separated energies, requiring a fixed $\rho+3 p=0$ equation of state.

Future work with the $R_{\mathrm{h}}=c t$ hypothesis still has much ground to cover. For example, the arguments we have made in this paper make a compelling case for the viability of this model, yet there is one more type of observation that supersedes all the rest: the actual measurement of the cosmic expansion rate as a function of redshift and/or time. In this vein, the so-called 'redshift drift' of sources moving in the Hubble flow has been recognized as a potentially powerful probe of the background cosmology. Estimates have shown that the first and second order redshift derivatives can be measured with upcoming surveys using ELT-HIRES [90] and the SKA Phase 2 array [91]. Even without a detailed calculation, one can intuitively see that an unambiguous prediction of the $R_{\mathrm{h}}=c t$ hypothesis is zero drift at all redshifts (see figure 9). This contrasts sharply with cosmologies, such as $\Lambda \mathrm{CDM}$, that predict a variable expansion rate.

Multi-year monitoring of objects at $z=5$ with the ELT-HIRES will show a velocity shift $\Delta v=-15 \mathrm{~cm} \mathrm{~s}^{-1} \mathrm{yr}^{-1}$ from the redshift drift in Planck $\Lambda$ CDM. By comparison, one expects $\Delta v=0 \mathrm{~cm} \mathrm{~s}^{-1} \mathrm{yr}^{-1}$ in $R_{\mathrm{h}}=c t$. The expected ELT-HIRES measurement error is $\pm 5 \mathrm{~cm} \mathrm{~s}^{-1}$ $\mathrm{yr}^{-1}$ after 5 years of monitoring. Therefore, these upcoming redshift drift measurements will differentiate between $R_{\mathrm{h}}=c t$ and Planck $\Lambda \mathrm{CDM}$ at a confidence level exceeding $3 \sigma$, so long as any possible source evolution is well understood. This is, of course, an important caveat. Assuming this hurdle can be overcome, such a result will provide the strongest evidence yet in favour of $R_{\mathrm{h}}=c t$. After 20 years of monitoring, this program of observations should favour one of these models relative to the others at better than $5 \sigma$ [88].

The reason this particular measurement is superior to all the rest is that, in the end, one does not even need to know the precise value of $\Delta v$, as long as one can demonstrate without any doubt that it is not zero. Any drift at all would completely obviate the viability of the $R_{\mathrm{h}}(t)=c t$ hypothesis and re-ignite the quest for understanding the (most remarkable) $R_{\mathrm{h}}\left(t_{0}\right)=c t_{0}$ coincidence in cosmology.

I am very grateful to Amherst College for its support through a John Woodruff Simpson 
Lectureship. I am also grateful to the Instituto de Astrofísica de Canarias in Tenerife and to

Purple Mountain Observatory in Nanjing, China for their hospitality while part of this work was carried out.

\section{References}

[1] W. Rindler Mon. Not. R. Astr. Soc. 116, 662 (1956).

[2] I. Ben-Dov Phys. Rev. D 75, id. 064007, 15pp (2007).

[3] V. Faraoni Phys. Rev. D 84, id. 024003, 15pp (2011).

[4] I. Bengtsson \& J.M.M. Senovilla Phys. Rev. D 83, id. 044012, 30pp (2011).

[5] V. Faraoni Cosmological and Black Hole Apparent Horizons, Springer, New York (2015).

[6] F. Melia Am. J. Phys. 86, 585 (2018).

[7] W. de Sitter Proc. Akad. Wetensch Amsterdam 19, 1217 (1917).

[8] A. Friedmann Zeitschrift für Physik 10, 377 (1923).

[9] F. Melia The Edge of Infinity-Supermassive Black Holes in the Universe, Cambridge University Press, Cambridge (2003).

[10] F. Melia MNRAS 382, 1917 (2007).

[11] F. Melia \& M. Abdelqader Int. J. Mod. Phys. D 18, 1889 (2009).

[12] G. Birkhoff Relativity and Modern Physics, Harvard University Press, Cambridge (1923).

[13] C. W. Misner \& D. H. Sharp Phys. Rev. 136, 571 (1964).

[14] W. C. Jr Hernandez \& C. W. Misner Astrophys. J. 143, 452 (1966).

[15] A. Prain, V. Vitagliano, V. Faraoni \& L. M. Lapierre-Léonard Class. Quantum Grav. 33, id. 145008, 13pp (2016).

[16] C. L. Bennett et al. Astrophys. J. Sup. 148, 1 (2003).

[17] D. N. Spergel et al. Astrophys. J. Sup. 148, 175 (2003).

[18] Planck Collaboration, P.A.R. Ade et al. Astron. E Astrophys. 571, A23, 48pp (2014).

[19] F. Melia Australian Phys. 49, 83 (2012). 
[20] F. Melia Frontiers of Phys. 11, id.118901 (2016).

[21] F. Melia Frontiers of Phys. 12, id.129802 (2017).

[22] F. Melia MNRAS 422, 1418 (2012).

[23] E. R. Harrison Astrophys. J. 446, 63 (1995).

[24] M. J. Chodorowski Mon. Notices R. Astr. Soc. 378, 239 (2007).

[25] M. J. Chodorowski Mon. Notices R. Astr. Soc. 413, 585 (2011).

[26] Yu. Baryshev Practical Cosmology, Proceedings of the International Conference "Problems of Practical Cosmology", held at Russian Geographical Society, 2008 in St. Petersburg, Edited by Yurij V. Baryshev, Igor N. Taganov, and Pekka Teerikorpi, (TIN, St.-Petersburg), 60 (2008).

[27] E. F. Bunn \& D. W. Hogg Am. J. Phys. 77, 688 (2009).

[28] R. J. Cook \& M. S. Burns Am. J. Phys. 77, 59 (2009).

[29] O. Gron \& O. Elgaroy Am. J. Phys. 75, 151 (2007).

[30] F. Melia \& A.S.H. Shevchuk Mon. Not. R. Astr. Soc. 419, 2579 (2012).

[31] Planck Collaboration et al. Astron. \& Astrophys. 594, id. A13 (2016).

[32] F. Melia Astrophys. Sp. Sc. 356, 393 (2015).

[33] J. R. Oppenheimer \& G. M. Volkoff Phys. Rev. 55, 374 (1939).

[34] G. C. McVittie Astrophys. J. 140, 401 (1964).

[35] I. H. Thompson \& G. F. Whitrow Mon. Not. R. Astr. Soc. 136, 207 (1967).

[36] S. Weinberg Gravitation and Cosmology: Principles and Applications of the General Theory of Relativity, Wiley, New York (1972).

[37] F. Melia Eur. Phys. Lett. in press (2019).

[38] F. Melia \& M. López-Corredoira Astron. 6 Astrophys. 610, id. A87 (2018).

[39] C. L. Steinhardt et al. Astrophys. J. 824, id. 21 (2016).

[40] M. K. Yennapureddy \& F. Melia Phys. Dark Univ. 20, 65 (2018).

[41] F. Melia Eur. Phys. J. C Lett. 78, id. 739 (2018).

[42] M. Fatuzzo \& F. Melia Astrophys. J. 846, id. 129 (2017). 
[43] F. Melia Europhysics Lett. 123, id. 39001 (2018).

[44] F. Melia \& M. K. Yennapureddy Mon. Not. R. Astr. Soc. 480, 2144 (2018).

[45] J.-J. Wei, X.-F. Wu \& F. Melia Mon. Not. R. Astr. Soc. 463, 1144 (2016).

[46] K. Leaf \& F. Melia Mon. Not. R. Astr. Soc. 474, 4507 (2018).

[47] F. Melia \& M. López-Corredoira Int. J. Mod. Phys. D 26, id. 1750055 (2017).

[48] H. Zeng, F. Melia \& L. Zhang Mon. Not. R. Astr. Soc. 462, 3094 (2016).

[49] M. López-Corredoira, F. Melia Int. J. Mod. Phys. D 25, id. 1650060 (2016).

[50] F. Melia Proc. R. Soc. A 472, id. 20150765 (2016).

[51] F. Melia \& R. S. Maier Mon. Not. R. Astr. Soc. 432, 2669 (2013).

[52] F. Melia \& T. M. McClintock Astronom. J. 150, id. 119 (2015).

[53] H. Yu \& F. Y. Wang Eur. Phys. J. C 74, id. 3090 (2014).

[54] F. Melia Astrophys. J. 764, id. 72 (2013).

[55] F. Melia Astron. E Astrophys. 615, id. A113 (2018).

[56] F. Melia \& T. M. McClintock Proc. R. Soc. A 471, id. 20150449 (2015).

[57] F. Melia Astrophys. Sp. Sci. 359, 34 (2015).

[58] J.-J. Wei, X. Wu, F. Melia, F.-Y. Wang \& H. Yu Astron. J. 150, id. 35 (2015).

[59] J.-J. Wei, X. Wu \& F. Melia Astron. J. 149, id. 165 (2015).

[60] J.-J. Wei, X. Wu, F. Melia \& R. S. Maier Astron. J. 149, id. 102 (2015).

[61] J.-J. Wei, X. Wu \& F. Melia Mon. Not. R. Astr. Soc. 447, 479 (2015).

[62] F. Melia Astron. J. 149, id. 2 (2015).

[63] K. Leaf \& F. Melia Mon. Not. R. Astr. Soc. 478, 5104 (2018).

[64] J.-J. Wei, X. Wu \& F. Melia Astrophys. J. 788, id. 190 (2014).

[65] F. Melia Astron. J. 147, id. 120 (2014).

[66] J.-J. Wei, X. Wu, F. Melia, D.-M. Wei \& L.-L. Feng Mon. Not. R. Astr. Soc. 439, 3329 (2014).

[67] F. Melia J. Cos. Part. Phys. 2014, id. 027 (2014). 
[68] J.-J. Wei, X. Wu \& F. Melia Astrophys. J. 772, id. 43 (2013).

[69] A. R. Liddle Mon. Not. R. Astr. Soc. Lett. 377, L74 (2007).

[70] J. E. Cavanaugh Aust. N. Z. J. Stat. 46, 257 (2004).

[71] G. Schwarz Ann. Statist. 6, 461 (1978).

[72] R. K. Sheth, H. J. Mo \& G. Tormen Mon. Not. R. Astr. Soc. 323, 1 (2001).

[73] S. G. Murray, C. Power \& A.S.G. Robotham Astron. \& Comp. 3, 23 (2013).

[74] F. Melia Mon. Not. R. Astr. Soc. 464, 1966 (2017).

[75] Planck Collaboration Astron. Es Astrophys. 571, id. A12 (2014).

[76] F. Melia Class. and Quant. Grav. 34, id. 015011 (2017).

[77] C. L. Bennett, D. Larson, J. L. Weiland et al. Astrophys. J. Sup. 208, id. 20 (2013).

[78] C. J. Copi et al. Mon. Not. R. Astr. Soc. 451, 2978 (2015).

[79] J. Kim \& P. Naselsky Astrophys. J. 739, id. 79 (2011).

[80] F. Melia Astron. \& Astrophys. 561, id. A80 (2014).

[81] A. Gruppuso et al. Phys. Dark Univ. 11, 68 (2016).

[82] F. Melia Astronom. \& Astrophys. 553, id.A76 (2013).

[83] A. H. Guth Phys. Rev. D 23, 347 (1981).

[84] A. Linde Phys. Lett. B 108, 389 (1982).

[85] A. Ijjas, P. J. Steinhardt \& A. Loeb PLB 723, 261 (2013).

[86] A. Ijjas, P. J. Steinhardt \& A. Loeb PLB 736, 142 (2014).

[87] F. Melia \& M. Fatuzzo Mon. Not. R. Astr. Soc. 456, 3422 (2016).

[88] F. Melia MNRAS Letters 463, L61 (2016).

[89] C.J.A.P. Martins, M. Martinelli, E. Calabrese \& M.P.L.P. Ramos Phys. Rev. D 94, id. 043001 (2016).

[90] J. Liske et al. Mon. Not. R. Astr. Soc. 386, 1192 (2008).

[91] H.-R. Klockner, D. Obreschkow, C.J.A.P. Martins, A. Raccanelli, D. Champion, A. L. Roy, A. Lobanov, J. Wagner \& R. Keller Proceedings, Advancing Astrophysics with the Square Kilometre Array (AASKA14), PoS AASKA14, 027 (2015). 